\title{
Hipertireoidismo Relacionado à Síndrome de McCune Albright: Relato de Dois Casos e Revisão da Literatura
}

\section{apresentação de caso}

\author{
ana Carolina R. Sallum \\ FERNANDO D. LEONHARDT \\ Onivaldo Cervantes \\ MÁrcio ABRAHĀo \\ REINALDO K. YAZAKI
}

Departamento de

Otorrinolaringologia e Cirurgia de Cabeça e Pescoço da Universidade Federal de São Paulo (Unifesp), SP, Brasil.

Recebido em 12/03/2007 Aceito em 05/12/2007

\begin{abstract}
RESUMO
Introdução: A síndrome de McCune Albright é uma doença rara, clinicamente definida pela combinação de displasia fibrosa poliostótica, manchas café-comleite e endocrinopatias com hiperfunção, como puberdade precoce, hipertireoidismo, acromegalia e outras. A fisiopatologia biomolecular baseia-se em uma mutação ativadora do gene para a subunidade alfa da proteína de membrana Gs, que estimula a produção intracelular de AMPc, conferindo secreção autônoma à glândula em particular. A glândula tireóide é freqüentemente envolvida nesta doença, sendo o hipertireoidismo a segunda endocrinopatia mais comum após a puberdade precoce. Objetivo: Realizar uma revisão da literatura no período de 1937 a 1997, incluindo nossa casuística, e discutir o tratamento utilizado para o hipertireoidismo nesta síndrome. Resultados: Do total de 85 casos, em 26 com hipertireoidismo se descreve o tipo de tratamento utilizado. Entre esses, 15 foram submetidos à cirurgia (tireoidectomia total), seis foram submetidos à iodoterapia e cinco foram submetidos a tratamento com drogas antitireoidianas (DAT - propiltiuracil, carbimazol ou metimazol), pois em alguns houve associação dos tratamentos citados anteriormente. Conclusão: A síndrome de McCune-Albright é uma doença rara, que inclui displasia fibrosa poliostótica, manchas café-com-leite e endocrinopatias com hiperfunção. Os casos acompanhados com hipertireoidismo são inicialmente tratados clinicamente com DAT, porém a ablação cirúrgica ou com radioiodoterapia é a opção definitiva mais indicada. (Arq Bras Endocrinol Metab 2008; 52/3:556-561)
\end{abstract}

Descritores: Hipertireoidismo; Síndrome; McCune-Albright; Endocrinopatia

\section{ABSTRACT}

Hyperthyroidism Related to McCune-Albright Syndrome: Report of Two Cases and Review of the Literature.

Introduction: McCune-Albright syndrome is a sporadic disease clinicaly characterized by polyostotic fibrous dysplasia, "café-au-lait" cutaneous spots and hyperfunctional endocrinopathies, such as precocious puberty, hyperthyroidism, acromegaly and others. The biologic physiopathology of the disease is based on an activating mutation of the gene for the Gs protein which mediates the activation of adenyl cyclase and subsequent gland autonomous secretion. The thyroid gland is usually involved in this disease, being hyperthyroidism the second most common endocrinopathy seen after precocious puberty. Objective: Revision of the literature since 1937 to 1997 , adding our casuistic and discussing the adequate treatment for the hyperthyroidism in the syndrome. Results: Among 85 cases identified with the syndrome, hyperthyroidism treatment was described in 26 of them. Fifteen were submitted to surgery (total thyroidectomy), 6 were submitted to iodotherapy and 5 were treated with antithyroid drugs (ATD - propylthiouracil, carbimazole or methimazole). In some cases, treatment association was done. Conclusion: McCune-Albright syndrome is a rare disease, combining polyostotic fibrous dysplasia, "café-au-lait" cutaneous spots and hyperfunctional endocrinopathies. Cases with hyperthyroidism are treated iniatially clinically, but definitive ablation with surgery or radioiodine treatment is the most indicated option. (Arq Bras Endocrinol Metab 2008; 52/3:556-561)

Keywords: Hyperthyroidism; Syndrome; McCune-Albrigt; Endocrinopathy 


\section{INTRODUÇÃO}

Síndrome de McCune-Albright é uma doença genética rara definida pela tríade de displasia fibrosa poliostótica, manchas cutâneas café-com-leite e endocrinopatias hiperfuncionantes, como puberdade precoce, hipertireoidismo e outras (1). Essa entidade foi descrita pela primeira vez por McCune e Albright em 1936 e 1937, respectivamente $(2,3)$.

A sua incidência ainda não é totalmente esclarecida, ocorrendo igualmente em todas as raças. A puberdade precoce gonadotrofina-independente é mais comum ocorrer no sexo feminino, enquanto as demais manifestações da síndrome de McCune-Albright ocorrem igualmente em ambos os sexos. Em relação à idade de aparecimento dos sintomas, nos casos mais graves envolvendo múltiplas endocrinopatias, a síndrome pode ser reconhecida logo após o nascimento e nos casos menos graves, os sintomas podem ser identificados em qualquer fase da infância (4), sendo geralmente mais precoces no sexo feminino (5).

A síndrome de McCune-Albright é o resultado de uma mutação do tipo mosaico que ocorre precocemente em estágio embrionário (6). A fisiopatologia fundamenta-se em uma mutação ativadora do gene para a subunidade alfa da proteína Gs, que estimula a produção intracelular de AMPc, conferindo secreção autônoma de tecidos endócrinos, gônadas, tireóide e adrenal (7). Essa mutação ativadora da proteína Gs também é demonstrada nas lesões cutâneas café-comleite e nas lesões ósseas da displasia fibrosa (7).

A apresentação clínica da doença é altamente variável, dependendo dos componentes mais predominantes na síndrome. Em casos em que a displasia fibrosa poliostótica seja marcante, múltiplas fraturas podem ser observadas logo no início do quadro, principalmente na infância. Outras endocrinopatias relatadas na síndrome de $\mathrm{McCu}-$ ne-Albright, além da puberdade precoce, incluem: bócio com ou sem hipertireoidismo, diabetes melito, acromegalia, síndrome de Cushing, hiperparatireoidismo, hiperprolactinemia e ginecomastia. Os distúrbios da tireóide são encontrados em $30 \%$ a $40 \%$ dos pacientes portadores da síndrome, sendo a incidência dessas lesões maior no sexo masculino (8). O hipertireoidismo é a segunda endocrinopatia mais comum da doença $(9)$.

O diagnóstico da doença é realizado pela correta identificação do quadro clínico e por meio de exames laboratoriais, que incluem a dosagem de hormônios sexuais, tireoidianos, cortisol e hormônio de crescimen- to. Exames de imagem, como ultra-sonografia pélvica (para pesquisa de cistos ovarianos), radiografia e cintilografia óssea (rastreamento ósseo) e tomografia computadorizada de abdome (para avaliar eventual aumento de volume das glândulas adrenais), podem auxiliar na confirmação do diagnóstico (4).

Os diagnósticos diferenciais da doença incluem todas as endocrinopatias que podem estar associadas à síndrome, como gigantismo e acromegalia, síndrome de Cushing, hipertireoidismo, neurofibromatose, puberdade precoce de origem central ou tumor ovariano funcionante (10).

O tratamento é direcionado para cada um dos elementos presentes na síndrome. A puberdade precoce na síndrome de McCune-Albright é gonadotrofina-independente e por isso não responde tão bem à terapia com agonista de hormônio liberador de gonadotrofina $(\mathrm{GnRH})$. Inibidores da aromatase, como testolactona, têm sido a principal terapia em meninas com elevação persistente de estradiol. Não é recomendado o tratamento cirúrgico para a puberdade precoce nessa síndrome (11).

Em casos de fibrodisplasia óssea sintomática, tem-se usado bifosfonados com o objetivo de aliviar a dor óssea. A fratura é a principal indicação de tratamento cirúrgico em casos de lesões displásicas. A maioria das fraturas é tratada com tração, entretanto, em fratura proximal do fêmur, opta-se pela fixação cirúrgica (1).

O hipertireoidismo, por causa das adenomas funcionantes da tireóide, pode ser tratado inicialmente com medicações antitireoidianas, como propiltiuracil e metimazol. O tratamento definitivo do hipertireoidismo inclui a terapia ablativa com ${ }^{131}$ I ou cirurgia (4).

Não existe um tratamento clínico efetivo para a síndrome de Cushing ACTH-independente e o tratamento recomendado nesses casos é a adrenalectomia bilateral, após a qual os pacientes necessitam de reposição de glicocorticóide e mineralocorticóide em quantidades apropriadas (4).

Em casos de gigantismo/acromegalia, o tratamento clínico com análogos da somatostatina é o ideal para adenomas produtores de $\mathrm{GH}$. O octreotide é um análogo da somatostatina de longa ação que tem sido usado com sucesso variável para reduzir a secreção de GH por esses tumores. A radioterapia não é recomendada nesses casos, pois pode causar alterações sarcomatosas em sítios ósseos onde haja displasia fibrosa. O tratamento cirúrgico só deve ser considerado se o tumor for visível e ressecável (4). 
É importante estimular os pacientes a manterem uma programação regular de exercícios físicos. Entretanto isso nem sempre é possível em pacientes com quadro grave de displasia fibrosa poliostótica (4).

A morbimortalidade da doença está associada a fraturas, malignidades, distúrbios endócrinos e outras condições associadas a essa síndrome. Apesar de um pequeno subgrupo desses pacientes possuir elevada mortalidade perioperatória e de desenvolver malignidades, a síndrome de McCune-Albright não está associada a aumento do risco de mortalidade. As deformidades relacionadas à displasia fibrosa poliostótica resultam diferentes graus de morbidade, desde casos leves a muito graves (4).

Não existem medidas preventivas para a síndrome de McCune-Albright, entretanto cuidados apropriados devem ser tomados na prevenção de fraturas em pacientes com displasia fibrosa poliostótica grave (4).

O objetivo desse estudo consiste na revisão da literatura acrescentando nossa própria casuística e discutir o tratamento utilizado para o hipertireoidismo na síndrome de McCune-Albright.

\section{PACIENTES E MÉTODOS}

Relatamos dois casos da síndrome de McCune-Albright com hipertireoidismo pertencentes ao nosso serviço e fizemos uma revisão da literatura de todos os casos publicados da associação da síndrome de McCune-Albright com distúrbios da tireóide (10-47).

\section{RELATO DE CASOS}

\section{Primeiro caso}

Paciente de 10 anos de idade, sexo feminino, com diagnóstico de síndrome de McCune-Albright após investigação de uma fratura patológica de fêmur associada a manchas cutâneas café-com-leite, puberdade precoce e hipertireoidismo. Ao exame físico apresentava bócio difuso, taquicardia e hiperatividade. Provas de função tireoidiana mostraram TSH suprimido $(<0,1 \mathrm{IU} / \mathrm{mL}) \mathrm{e}$ aumento da concentração sérica de T4 livre $(2,5 \mathrm{ng} /$ $\mathrm{dL}$ ). TRAB (thyrotrophin receptor antibody) e anticorpos antiperoxidase encontravam-se negativos. Houve insucesso no tratamento clínico do hipertireoidismo e foi realizada uma tireoidectomia total.

\section{Segundo caso}

Paciente de 6 anos de idade, sexo feminino, que apresentava palpitações, taquipnéia e dor torácica. Displasia óssea poliostótica, manchas cutâneas café-com-leite e puberdade precoce estavam associadas com hipertireoidismo. $\mathrm{O}$ exame físico revelou taquicardia, sopro sistólico na área cardíaca, deformidades ósseas e bócio difuso. Provas de função tireoidiana mostraram TSH suprimido $(<0,1 \mathrm{IU} / \mathrm{mL})$ e aumento da concentração sérica de T4 livre $(5,4 \mathrm{ng} / \mathrm{dL})$. TRAB e anticorpos antiperoxidase também negativos. Após falha do tratamento clínico, optou-se também por realizar tireoidectomia total.

\section{RESULTADOS}

Em pesquisa bibliográfica da literatura a partir de 1937 - ano em que a síndrome foi descrita pela primeira vez por McCune e Albright - a 1997, foram descritos 83 casos da síndrome de McCune-Albright associados a tireoideopatias. Com os dois casos relatados em nosso serviço, totalizam-se 85 casos desta associação.

Entre os distúrbios da tireóide encontrados observou-se 55 pacientes $(65 \%)$ com hipertireoidismo clínico, 12 (14\%) com hipertireoidismo subclínico, seis (7\%) com bócio difuso e cinco (6\%) com bócio nodular, ressaltando-se que em sete pacientes não foi caracterizado o tipo de tireoidopatia (8\%).

A idade de início dos sintomas variou desde recémnato (quatro dias de vida) até 49 anos de idade.

Dos 83 pacientes, apenas em 26 foi descrito o tipo de tratamento utilizado, incluindo nossos dois pacientes. Entre esses, 15 (57\%) foram submetidos à cirurgia (tireoidectomia total), seis (23\%) foram submetidos à iodoterapia e cinco $(20 \%)$ foram submetidos a tratamento apenas com DAT (propiltiuracil, carbimazol ou metimazol). Em alguns pacientes houve combinação dos tratamentos citados anteriormente. Após o tratamento todos os pacientes apresentaram melhora clínica do quadro de hipertireoidismo.

Apenas em cinco dos 83 pacientes relatados foi descrita a mutação genética responsável pela síndrome. Nestes, a mutação consistiu na substituição da arginina na posição 201 da subunidade alfa da proteína Gs pelo aminoácido cisteína (quatro pacientes) ou histidina (um paciente). 


\section{DISCUSSÃo}

A extensa análise da literatura confirmou que os distúrbios da tireóide consistem na segunda endocrinopatia mais comum na síndrome de McCune-Albright, visto que essas tireoidopatias variaram desde hipertireoidismo subclínico até hipertireoidismo franco com bócio. Nesse estudo, o distúrbio tireoidiano mais freqüente foi o hipertireoidismo clínico (65\%), no qual os pacientes apresentavam sinais e sintomas associados à elevação do nível dos hormônios tireoidianos.

De todas as endocrinopatias dessa síndrome a puberdade precoce é a mais comum, seguida por distúrbios tireoidianos, encontrados em 30\% a $40 \%$ dos pacientes portadores da síndrome (6), aumento dos níveis de hormônio do crescimento resultando acromegalia, hiperprolactinemia, hipercortisolismo e hiperparatireoidismo (34).

A síndrome de McCune-Albright é resultado de uma mutação do tipo mosaico que ocorre precocemente em estágio embrionário (6). Essa mutação genética consiste na substituição da arginina na posição 201 da subunidade alfa da proteína Gs pelo aminoácido cisteína ou histidina, inibindo assim a atividade da enzima guanosina trifosfatase e levando a uma prolongada estimulação da adenilciclase e conseqüente aumento da produção de AMP cíclico, resultando hiperfunção dos tecidos afetados, como o tireoidiano, o gonadal, o adrenal, a pele e o osso (4)

Conforme demonstrado nesta pesquisa, a idade de aparecimento dos sintomas é bastante variável, dependendo da gravidade do caso, pois casos mais graves podem ser identificados mais precocemente que os menos graves. Da mesma forma, a apresentação clínica da doença é altamente variável, dependendo dos vários componentes predominantes na síndrome. Em casos em que a displasia fibrosa poliostótica seja marcante, múltiplas fraturas podem ser observadas logo no início do quadro, principalmente na infância. A displasia fibrosa poliostótica consiste na substituição de osso normal por tecido ósseo fibroso imaturo e desorganizado em razão do supercrescimento de células mesenquimais primitivas, isto é, aumento do número de células progenitoras osteogênicas levando à formação de áreas fibróticas no osso, causando dor, fragilidade e deformidades ósseas (1).

O tratamento é direcionado para cada um dos elementos presentes na síndrome. No caso do hipertireoi- dismo, medicações antitireoidianas podem ser usadas inicialmente para tentar controlar o quadro clínico e laboratorial. Confirmando-se o diagnóstico da síndrome, deve-se priorizar um tipo de tratamento mais definitivo do hipertireodismo, como terapia ablativa com ${ }^{131}$ Iodo ou tireoidectomia total, que nesse estudo constituiu de terapia de escolha em $84 \%$ dos casos em que foi descrito o tratamento. Recomenda-se o tratamento cirúrgico definitivo (tireoidectomia total) ou a ablação completa da glândula com ${ }^{131}$ I para o hipertireoidismo na síndrome de McCune-Albright, uma vez que os sintomas de hipertireoidismo devem recorrer após a retirada dos medicamentos antitireoidianos, dado que se trata de uma mutação constitutiva (6).

Em ambos os casos pertencentes ao nosso serviço, a despeito da idade dos pacientes, optou-se pela tireoidectomia total com o objetivo de evitar a recorrência dos sintomas e de manter um melhor controle clínico do quadro de hipertireoidismo a longo prazo, uma vez que já havia ocorrido insucesso com o tratamento clínico.

O hipertireoidismo com ou sem bócio no contexto da síndrome de McCune-Albright é considerado uma causa rara, porém bem descrita, de disfunção tireoidiana não auto-imune, sendo considerada a segunda endocrinopatia mais comum na síndrome, conforme observado na literatura.

É importante que os médicos que estejam diante de um quadro de hipertireoidismo sejam capazes de identificar se há outras manifestações clínicas e associálas à síndrome de McCune-Albright. O diagnóstico precoce dessa síndrome é essencial para iniciar o tratamento no momento adequado.

A síndrome de McCune-Albright é uma condição endocrinológica rara, cujos casos com hipertireoidismo podem ser inicialmente tratados clinicamente com DAT. A ablação cirúrgica ou com iodo radioativo são as opções mais indicadas para o tratamento definitivo do hipertireoidismo.

\section{REFERÊNCIAS}

1. Riminucci M, Fisher LW, Shenker A, Spiegel AM, Bianco P, Robey PG. Fibrous dysplasia of bone in the McCune-Albright syndrome. Abnormalities in bone formation. Am $\mathrm{J}$ Pathol. 1997;151(6):1587-600.

2. McCune DJ, Bruch H. Osteodystrophia fibrosa: report of a case in which condition was combined with precocious puber- 
ty, pathologic pigmentation of skin and hyperthyroidism, with a review of the literature. Am J Dis Child. 1937;54:806-48.

3. Albright F, Scoville B, Sulkowitch HW. Syndrome characterized by osteitis fibrosa disseminata, areas of pigmentation and a gonadal dysfunction. Further observations including report of two more cases; discussion by R. Barnwell, patient of A. H. Musser. Endocrinology. 1938;22:411-21.

4. Shenker A, Weinstein LS, Moran A, et al. Severe endocrine and nonendocrine manifestations of the McCune-Albright syndrome associated with activating mutations of stimulatory G protein GS. J Pediatr Oct. 1993;123(4):509-18.

5. Cohen MM Jr, Howell RE. Etiology of fibrous dysplasia and McCune-Albright syndrome. Int J Oral Maxillofac Surg. 1999;28(5):366-71.

6. Shenker A, Weinstein LS, Sweet DE. An activating Gs alpha mutation is present in fibrous dysplasia of bone in the McCune-Albright syndrome. J Clin Endocrinol Metab. 1994;79(3):750-5.

7. Weinstein LS, Shenker A, Gejman PV, Merino MJ, Friedman E, Spiegel AM. Activating mutations of the stimulatory $G$ protein in the McCune-Albright syndrome. $N$ Engl J Med. 1991;325:1688-95.

8. Mastorakos G, Mitsiades NS, Doufas AG, Koutras DA. Hyperthyroidism in McCune-Albright syndrome with a review of thyroid abnormalities sixty years after the first report. Thyroid. 1997;7:433-9.

9. Cavanah SFW, Dons RF. McCune-Albright syndrome: how many endocrinopathies can one patient have? South Med J. 1993;86(3):364-7.

10. de Sanctis C, Lala R, Matarazzo P, et al. Pubertal development in patients with McCune-Albright syndrome or pseudohypoparathyroidism. J Pediatr Endocrinol Metab. 2003;16 Suppl 2:293-6.

11. Feuillan PP, Foster $\mathrm{CM}$, Pescovitz $\mathrm{OH}$, et al. Treatment of precocious puberty in the McCune-Albright syndrome with the aromatase inhibitor testolactone. N Engl J Med. 1986;315(18):1115-9.

12. Mauras N, Blizzard RM. The McCune-Albright syndrome. Acta Endocrinol Suppl. 1986;279:207-17.

13. Peck FB, Sage CV. Diabetes mellitus associated with Albright's syndrome. Am J Med Sci. 1944;208:35.

14. Jervis GA, Schein H. Polyostotic fibrous dysplasia (Albright's syndrome). Arch Pathol. 1951;51:640-50.

15. Yettra M, Starr P. Polyostotic fibrous dysplasia associated with hyperthyroidism. J Clin Endocr. 1951;11: 312-21.

16. Sternberg WH, Joseph V. Osteodystrophia fibrosa combined with precocious puberty and exophthalmic goiter. Am J Dis Child. 1952;63:748-83.

17. Vague J, Marcorelles J, Coulomb J. Dysplasie fibreuse de os, puberté précose, maigreur merveuse et hyperthyréose. Ann Endocrinol. 1958;19:1169-73.

18. Halvorsen S, Aas K. Renal tubular defects in fibrous dysplasia of the bones. Reports of two cases. Acta Pediatr. 1961;50:297.

19. Kim JP, Khera SAK. Polyostotic fibrous dysplasia associated with hyperthyroidism. J Bone Joint Surg. 1961;43-A:897-904.

20. Benedict $P$. Endocrine features in Albright's syndrome (fibrous dysplasia of bone). Metabolism. 1962;11:30-45.

21. Moldawer M, Rabin ER. Polyostotic fibrous dysplasia with thyrotoxicosis. Arch Intern Med. 1966;118:379-84.

22. Zangeneh F, Lulejian GA, Steiner MM. McCune-Albright syndrome with hyperthyroidism. Am J Dis Child. 1966;111:644-8.
23. Firat D, Stutzman L. Fibrous dysplasia of bone. Review of twenty-four cases. Am J Med. 1968;44:421-3.

24. Samuel S, Gilman S, Maurer HS, Rosenthal IM. Hyperthyroidism in an infant with McCune-Albright syndrome: report of a case with myeloid metaplasia. J Pediatr. 1972;80:275-8.

25. Hamilton CR Jr, Maloof F. Unusual types of hyperthyroidism. Medicine. 1973;52:195-215.

26. Andrews SB, Posen S. Thyrotrophin in Albright's syndrome with hyperthyroidism [letter to the editor]. Ann Intern Med. 1974;81:561.

27. Tanaka T, Suwa S. A case of McCune-Albright syndrome with hyperthyroidism and vitamin D-resistant rickets. Helv Pediatr Acta. 1977;32:263-73.

28. Giovanelli G, Bernasconi S, Banchini G. McCune-Albright syndrome in a male child: a clinical and endocrinologic enigma. $J$ Pediatr. 1978;92 Suppl 2: 220-6.

29. Richton SM, Maclaren NL, McLaughlin JV, Raiti S. Albright's syndrome presenting as thyrotoxicosis: follow up of a case. Pediatrics. 1979;63:159-62.

30. Albin J, Wu R. Abnormal hypotalamic-pituitary function in polyostotic fibrous dysplasia. Clin Endocrinol (Oxf). 1981;14:435-43.

31. D'Armiento M, Reda G, Camagna A, Tardella L. McCune-Albright syndrome: evidence for autonomus multiendocrine hyperfunction. J Pediatr. 1983;102:584-6.

32. Domenech E, Santisteban M, Moya M, Gonzalez C, Cortabarria C, Mendez A, et al. Transient neonatal hyperthyroidism in the child of a treated hyperthyroid mother. Subsequent appearence of sexual precocity. An Esp Pediatr. 1985;22:281-7.

33. Lee PA, Van Dop C, Migeon C. McCune-Albright syndrome, long-term follow-up. JAMA. 1986;256 Suppl 1:2980-4.

34. Summerfeldt $P$, Brown A. Osteodystrophia fibrosa. Am J Dis Child. 1939;57:90.

35. Misaki M, Shima T, Ikoma J, Morioka K, Suzuki S. Acromegaly and hyperthyroidism associated with McCune-Albright syndrome. Horm Res. 1988;30:26-7.

36. Gallacher SJ, Wilson R, Boyle IT, Thompson JA. The hyperthyroidism of polyostotic fibrous dysplasia - A possible autoimmune etiology. Scottish Med J. 1989;34:529.

37. Huang TS, Chang CC, Huang JS, Hsieh HC, Chen FW. Albright's syndrome with acromegaly and Hashimotos's thyroiditis: report of a case. J Formosan Med Assoc. 1990;89:714-8.

38. Feuillan PP, Shawker T, Rose SR, Jones J, Jeevanram RK, Nisula $B$. Thyroid abnormalities in the McCune-Albright syndrome: ultrasonography and hormonal studies. J Clin Endocrinol Metab. 1990;71:1596-1601.

39. Abs R, Beckers A, Van de Vyver FL, De Schepper A, Stevenaert A, Hennen G. Acromegaly, multinodular goiter and silent polyostotic fibrous dysplasia. A variant of the McCune-Albright syndrome. J Endocrinol Invest. 1990;13:671-5.

40. Yoshimoto $M$, Nakayama M, Baba T, Uehara T, Niikawa N, Ito $M$, et al. A case of neonatal McCune-Albright syndrome with Cushing syndrome and hyperthyroidism. Acta Paediatr Scand. 1991;80:984-7.

41. Lawless ST, Reeves G, Bowen JR. The development of a thyroid storm in a child with McCune-Albright syndrome after orthopedic sugery. Am J Dis Child. 1992;146:1099-102.

42. Mastorakos G, Modigliani E, Hassan M, Gaudouen Y, Krivitzky A. Acromegaly without pituitary adenoma in McCune-Albright syndrome, successfully treated with a somatostatin analog. J Pediatr Endocrinol. 1992;5:271-4. 
43. Schwindinger WF, Francomano CA, Levine MA. Identification of a mutation in the gene enconding the alpha subunity of the stimulatory $\mathrm{G}$ protein of adenylyl cyclase in McCune-Albright syndrome. Proc Natl Acad Sci USA. 1992;89:5152-6.

44. Lair-Milan F, Le Blevec G, Carel JC, Chaussain JL, Adamsbaum C. Thyroid sonographic abnormalities in McCune-Albright syndrome. Pediatr Radiol. 1996;26:424-6.

45. Brogan P, Khadilkar VV, Stannhope R. Occult T3 toxicosis in McCune-Albright syndrome. Horm Res. 1998;50:105-6.

46. Isotani H, Sanda K, Kameoka K, Takamatsu J. McCune-Albright syndrome associated with non-autoimmune type of hyperthyroidism with development of thyrotoxic crisis. Horm Res. 2000;53:256-9.

47. Leet Al, Chebli $\mathrm{C}$, Kushner $\mathrm{H}$, Chen CC, Kelly MH, Brillante BA, et al. Fracture incidence in polyostotic fibrous dysplasia and the McCune-Albright syndrome. J Bone Miner Res. 2004;19:571-7.
Endereço para correspondência:

Ana Carolina Raposo Sallum

Rua Cardoso de Almeida, 668, apto. 163 - Perdizes,

05013-000, São Paulo SP

Email: carolsallum@terra.com.br 